\title{
Photography in dentistry: A perspective
}

\begin{abstract}
Photography is an "informative tool" to evaluate the diagnosis, make the proper treatment plan, document cases, communicate with the dental technician and other specialties for a multidisciplinary approach. These all can be achieved by only a good photograph with illumination, sharpness, and proper color contrast. This article emphasizes on photography and technical aspects which are required to understand photography in the field of dentistry. By using accessories like photographic mirror, contrastors and retractors, we can record the details of a targeted area. The advances in techniques of photography gives good results provided one understands the complexity of the camera-system.
\end{abstract}

Volume 12 Issue 5 - 2020

\author{
Mithra N Hegde,' Urvashi B Sodvadiya ${ }^{2}$ \\ 'Vice Principal/ Head of the Department, Department of \\ Conservative Dentistry \& Endodontics,A.B.Shetty Memorial \\ Institute of Dental Sciences, India \\ ${ }^{2}$ Post-graduate student,ABSMIDS, Nitte (Deemed to be \\ University), India
}

\begin{abstract}
Correspondence: Mithra N Hegde MDS, PhD, MAMS, Vice Principal/ Head of the Department of Conservative Dentistry \& Endodontics, A.B.Shetty Memorial Institute of Dental Sciences, NITTE (deemed to be university), Mangalore- 575018, India, Tel+9I-98452844I I, Email drhgdedentist@gmail.com, mithrahegde@yahoo.co.in
\end{abstract}

Received:June 19,2019 | Published: November 26, 2020

\section{Introduction}

Photography plays a significant role in communication. The photographic image is the positive replica of an object.

Clinical photography in the field of dentistry is one of the evolutionary aspects of communication. From film camera to digital camera, improvement in many features like the quality of image and ease of use can be observed. ${ }^{1}$ Dental photography was pioneered by Dr. R. Thompson and W. Elde of Columbus (1948). ${ }^{2}$ More recently, use of photography for documentation of cases, presentation of the case,and patient education has increased, and it became an essential part of modern dentistry.

\section{Need for photography}

\section{Diagnosis and treatment planning}

A series of photographs before starting any treatment is the ideal method to evaluate the case and to make a baseline for an oral health condition for the patient. Based on that, a treatment plan can be made easily,just like study cast arebeing used for the treatment planning of an orthodontic case. ${ }^{3}$ It is advised to click a series of different views of extraoral and intraoral photographs. Extraoral photographs include full face (frontal \& lateral, with different expression), full natural smile (frontal \& lateral), lips at rest position while intraoral photographs include occlusal photograph (maxillary and mandibular arch), full dentition retracted view (teeth slightly apart and closed), any specialview (photograph of any particular tooth). ${ }^{4}$

\section{Case documentation}

Documentation of the patient's initial condition is beneficial to refer the case on a later date. In the initialexamination, many things remained undiagnosed. But a proper series of photographs will help you to disclose these overlooked findings. ${ }^{1}$

\section{Communication}

In aesthetic dentistry, images play a vital role incommunication with dental technician and patients as well. For laboratory communication, it will not just provide the information about shade but will also give an idea about contour, contact, form of the tooth,and translucency of enamel. ${ }^{5}$

A better understanding of each step of treatment to the patient can be best achieved by photographs. Apart from it, this will help in building a healthy relationship between doctor and patient. ${ }^{6}$

\section{Education}

Photography is an "informative tool" not only for treatment planning and diagnosis,but it is also helpful for self-assessment and self-education. A case which requires a multi-disciplinary approach, photography will help in collaboration with different specialty. ${ }^{7}$ Apart from this, digital photography can be used in forensic dentistry, insurance verification, marketing, and documenting for scientific research and publication. Before taking any photographs, it is necessary to take consent of the patient.

\section{What do we look for in positive replica?}

Intraoral photography is one kind of macro-photography which allows you to take a photograph of an object in magnified view. The main problem with this type of photography is to get proper exposure for better illumination and depth of field for better clarity.

\section{Illumination}

All photographs require a proper light source while capturing. Best light source to click a photograph is natural daylight. There are different light sources available for photography like an electronic flash, quartz lighting, ultraviolet illumination,etc. Selection of light source should be in such a way that it should not hamper the quality of the image. ${ }^{8}$ It will be challenging to evaluate contour and to select the shade if improper light is used to click the photograph. ${ }^{9}$

\section{Sharpness and clarity}

Quality of the image can be measured by clarity and sharpness of the image. In dental photography, clarity of the image can be modified by changing the focusing plane or area. When we talk about dental photography, to click the image with better clarity, certain are as are determined for different views (Table 1$).^{10}$ 
Table I Where to focus to obtainmaximum depth of field for intra-oral photography?

\begin{tabular}{ll}
\hline Views & Focusing area \\
\hline Entire arch & Maxillary canine \\
Occlusal view & Premolar \\
Maxillary anteriors & Lateral incisor \\
\hline
\end{tabular}

\section{Colour}

An image with natural color is the best image one can capture. Different sources of light have different color content. It is described and measured as color temperature (degrees Kelvin) by the color scientist. For example, the color temperature of daylight is 5500-6500 $\mathrm{K}$ while for the cloudy sky, it is around $6500-8000 \mathrm{~K}$. If the color is different in an image,then the natural color of an object; it can lead to a miscommunication between a dentist and dental technician. Colour of an image can be modified by white balance. ${ }^{11}$

\section{Consistency}

For any case documentation, pre-operative, intra-operative, and post-operative photographs of each view are recommended. To evaluate the outcome of the treatment,consistency of each photograph should be maintained. To maintain the consistency, magnification ratio, and working distance should be maintained throughout the photography session. ${ }^{10,12}$

\section{Methods to achieve it}

\section{Exposure control}

When we pressed the "picture-taking button,"the light will pass through the aperture (small opening of the camera), and the sensor will get exposed to create an image. Exposure is the control of the amount of light falling on the sensor when a photographis clicked. If the amount of light is unnecessarily high, the resultant image will be overexposed and if it is too low, the resultant image will be underexposed. ${ }^{11}$ 2):

Exposure can be controlled by mainly three factors (Figures $1 \&$

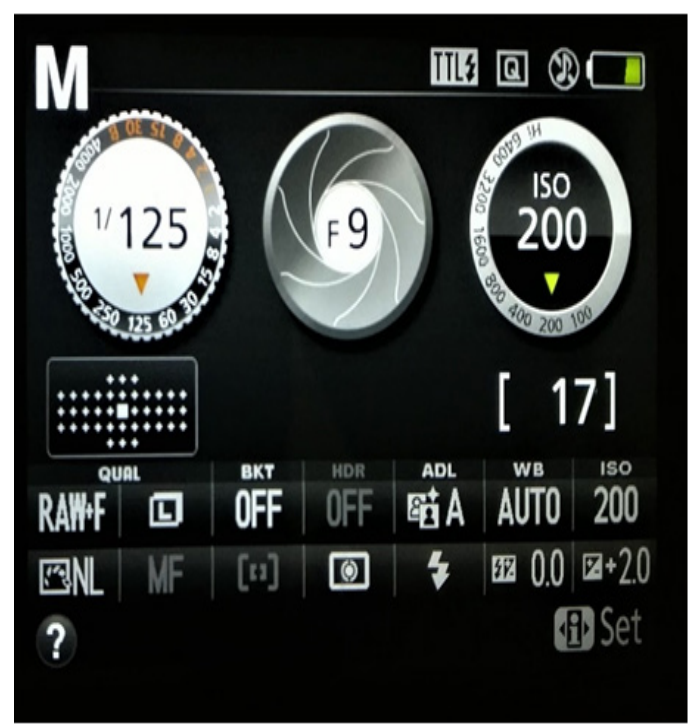

Figure I Setting mode of camera showing shutter speed, f-stop (size of aperture), sensitivity of sensor (ISO).

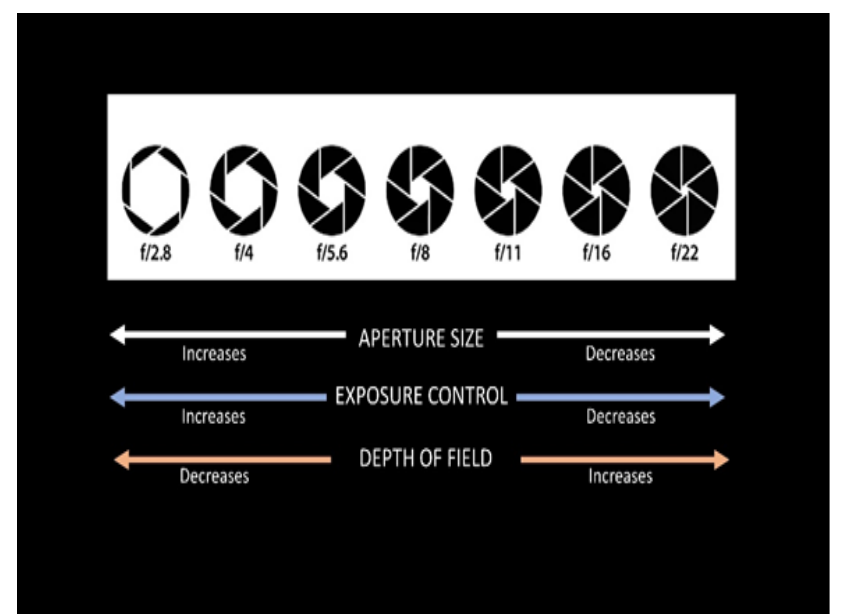

Figure 2 Relationship between size of aperture, exposure control and depth of field.

\section{a. Sensitivity of sensor}

ISO sensitivity is the designated as number (given by International Organization for Standardization) which is defined as the sensitivity of the sensor to light. If the ISO number is higher (for example, a camera with ISO 400), the sensitivity of the sensor to the light is higher (will require lesser light to produce an image).The lowerISO number is recommended to get a high quality, clear picture and to avoid grainy pictures. ${ }^{12}$

\section{b. Size of aperture}

Size of lens opening through which light passes is known as aperture. Size of the apertureis calibrated as f-Stop (f-Ratio) which is a ratio of focal length to size of aperture. $f-32$ is interpreted as the diameter of aperture is $1 / 32$ th of the focal length of the lens. f-stop is directly related to the depth of field..$^{13}$ Hence a camera with smallest aperture size (available smallest size is: $\mathrm{f}=32$ ) can click a photograph with the highest depth of field. But practically this opening is too small to allow light to pass through. Therefore, f 22 is recommended for dental photography. ${ }^{14}$

\section{c. Time of exposure}

Time of exposure can be controlled by shutter speed and amount of flash burst. Shutter speed is determined by the speed at which curtain slides off the sensor and after a given time sensor will be covered by another curtain and image exposure will stop. Shutter speed should not be either too slow to avoid blurring of the image due to the patient's movement or too fast to avoidthe darkness of image due to less exposure. ${ }^{15}$ For dental photography, shutter speed ranges from $1 / 60 \mathrm{~s}$ to $1 / 120 \mathrm{~s}^{14}$

\section{Depth of field}

Depth of field is definedas the zone of sharpness within a photo for which focus was made. Depth of field (clarity) is distributed in the image as follows (based on focused area):

i. $1 / 3^{\text {rd }}$ in front of focused area

\section{ii. $2 / 3^{\text {rd }}$ behind the focused area}

Depth of field is affected by the size of aperture and photography distance. With the decreased size of aperture, depth of field increases while photography distance is indirectly proportional to the depth of field $^{14}$ (Figure 2). 


\section{White balance}

White balance is one of the essential functions of the camera; by modifying this function, one can capture an image with natural color. We can easily differentiate an image with natural color and an image which may not reflect natural color. The different setting of white balance tries to modify the light and capture the image as natural as possible. Hence, it results in an image with more natural color reproduction.From all the different setting, flash white balance is recommended for dental photography (Figure 3). Hence, an auto mode for white balance is not suitable for dental photography. ${ }^{14}$

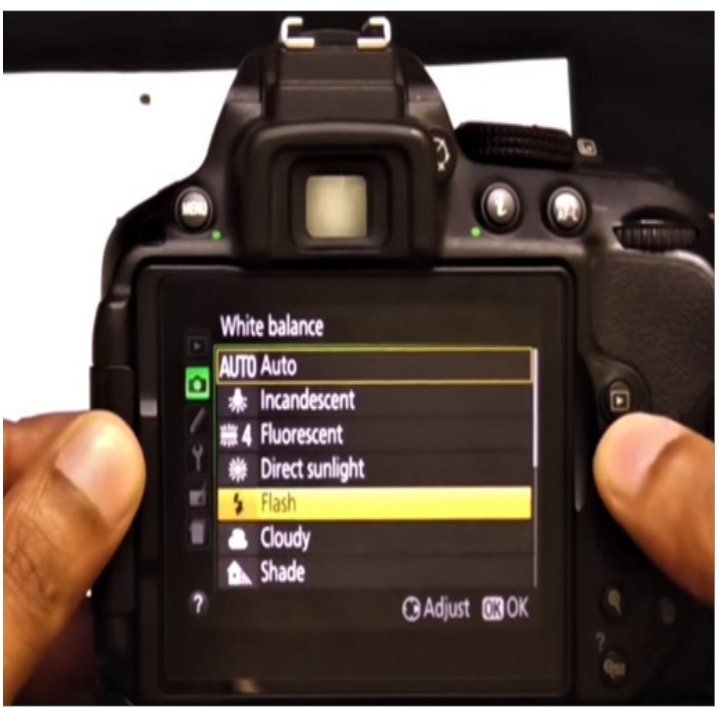

Figure 3 Setting mode for white balance.

\section{Magnification ratio}

The magnification ratio is a ratio of image size to object size This ratio can be affected by the size of the sensor. But nowadays, all cameras have $35 \mathrm{~mm}$ size film, so modification is not needed. The magnification ratio is affected by working distance. Working distance is the distance between the lens and an object. In recent lenses, for a given magnification ratio working distance is advised in the camera setting itself. Therefore, one can capture a consistent series of images. For different views, the different magnification ratio is advised (Table 2). ${ }^{10}$

Table 2 Recommended magnification ratio for different views

\begin{tabular}{ll}
\hline Views & Magnification ratio \\
\hline Portrait & $01: 10$ \\
Full dental arch- in occlusion(frontal view) & $0 \mathrm{I}: 02$ \\
Full dental arch- in occlusion (lateral view) & $0 \mathrm{I}: 0 \mathrm{I} .5$ \\
Anterior teeth (canine-canine; frontal view) & $0 \mathrm{I}: 0 \mathrm{I} .2$ \\
Occlusal view of arch (maxillary/mandibular) & $0 \mathrm{I}: 02$ \\
Maxillary central incisors & $\mathrm{I} .5: \mathrm{I}$ \\
\hline
\end{tabular}

\section{Number of pixels}

Pixel is the smallest unit that makes up an image (digital image). Quality of an image is not affected by number of pixels; it determines the size of an image. For instance, the 3-megapixel camera is enough to take a photograph of $5 \times 7$ inches. If the bigger size of photograph is required, then a camera with higher megapixel should be chosen.
Quality of an image is affected by white balance, the resolution power of the lens, color modification by the camera, a storage system of camera,etc. For dental photography minimum of the 6-megapixel camera is recommended, because the maximum size of A4 print is required to print out a dental photograph. However, most current cameras have a minimum of 10 megapixels as a standard. ${ }^{12}$

\section{Classification of camera}

Basic classification of the camera includes two types- still and action. Still, cameras are for photography while the other one is for videography. Initially,the filmwas used in cameras which must be chemically processed to get a print of the photograph. But now it is replaced by a digital sensor (charge coupled device- CCD or a complementary metal oxide semiconductor- CMOS). Modern cameras are divided into two categories- Rangefinder and Digital Single Lens Reflex camera (DSLRs). The primary difference between these two categories is the presence of parallax effect in Rangefinder cameras. ${ }^{16}$

In dentistry, Intraoral Fibre Optic Cameras, Instant Polaroid film Cameras, Kodak P712 Digital camera system (specifically manufactured for dentistry) and Compact cameras had been used. Nowadays, most commonly available cameras include DSLRs, pocket-sized cameras,and personal electronic gadgets (example; smartphones, tablets, and laptop). Among these three, DSLRs is highly recommended for dental photography. Asthe lens can be interchanged and so many functions can be manually modified in this category. While in pocket-sized cameras and cameras of personal electronic gadgets have inbuilt fixed lenses and have very few functions which can be adjusted manually. But most commonly used one is, cameras of personal electronic gadgetssince most of us carry at least one personalgadget along at all the time and it is easy to use, transfer and store. ${ }^{12}$

SLR cameras work on a mirror and prism system which allows the photographer to see Through-the-Lens (TTL) and capture the same image, which can be seen in the view finder. Technically, SLR and DSLR cameras work on the same principle, but few functions are different. For instance, SLR cameras have a film to record the image which must chemically be processed to get a photograph. As the film is being used for SLR cameras, storage capacity is deficient (maximum 35 films). While DSLR camera has a digital sensor, so it captures the images digitally (via digital sensor). A DSLR camera has a memory card so, replacement of memory card is not needed frequently. You can transfer all the images electronically and can reuse the same memory card later. Apart from these, both types of camera have a different attachment apparatus to which additional flash can be incorporated into photography. ${ }^{17}$

\section{Lenses}

A mostimportant component of these DSLR cameras is "Lens."Lensisavailable in different focal length. The focal length is the distance between the lens and sensor/film. The available focal length ranges from $35 \mathrm{~mm}$ to $200 \mathrm{~mm}$. For dental photography lens with 60-, $85-$ and $100-\mathrm{mm}$ focal length is recommended. Among these three, most recommended one is a lens with $100 \mathrm{~mm}$ focal length. ${ }^{17}$ Two types of lenses are available- general purpose lens and macro lenses. Difference between these two is, general purpose lens doesn't have as much focusing capability as macro lenses,and close-up photography is only possible with macro lenses. Moreover, general purpose lens doesn't have a feature of grids for consistency in the photograph. These lenses are used in pocket-sized cameras and personal electronic gadgets. ${ }^{13} \mathrm{~A}$ macro lens is suitable for dentistry, which can capture 
highly focused close-up photographs. Currently, 'Telephoto lens' is introduced,which is physically shorter in length than its actual focal length. Thisis accomplished by incorporating a lens in such a way that increases the path for light to create a long-focus length in a shorter design. Therefore, this lens is highly recommended for dental photography.

\section{Light and modification of light}

With a lack of light and presence of shadow, it is difficult to capture a high-quality photograph in dentistry. Especially for intraoral photography, flashes are the integralpart of a camera. Three types of flashes are available- single point, twin flash,and ring flash. These all flashes have their advantages and disadvantages, are recommended for different situations ${ }^{13,18}$ (Table 3 ).

Table 3 All flashes have their advantages and disadvantages, are recommended for different situations

\begin{tabular}{|c|c|c|}
\hline Flash type & Use & Advantages \&disadvantages \\
\hline Single point & - To capture anterior teeth (a small segment) & $\begin{array}{l}\text { - It creates a shadow so, the contour of anterior teeth can be readily appreciated } \\
\text { in the photographs. But it is not recommended for the entire arch. }\end{array}$ \\
\hline \multirow[t]{2}{*}{ Twin flash } & - To capture the entire arch & $\begin{array}{l}\text { - The second flash is used to cover the shadow which is created by using a single } \\
\text { point flash }\end{array}$ \\
\hline & $\begin{array}{l}\text { - For the attachment to camera, adjustable arr } \\
\text { - Can be used at different angulation. (45 degr }\end{array}$ & $\begin{array}{l}\text { sttachment is available. } \\
\text { s; one on each side is recommended) }\end{array}$ \\
\hline \multirow[t]{3}{*}{ Ring flash } & - To capture the entire arch & - It emits too much light. \\
\hline & - It is attached to the front end of the lens & Therefore, the capturedimage is flat and bland (due to the absence of shadow) \\
\hline & & - Not recommended to capture an image for anterior teeth \\
\hline
\end{tabular}

This light can be modified by blocking, diffusing,and using reflectors. In twin flash, if twin flash is not required, one flash can be blocked, and just a singleflash can be used to capture an image. Light can be diffused by using a material (for example, a cloth of different opacities) in front of light to decrease the intensity and amount of falling light on to the object. Various types of reflectors are available in the market which is placed in the opposite direction of the primary light source. Deflected light can be used in the diagnosis of enamel cracks and dentin characteristics. ${ }^{11}$

\section{Accessories required for intra-oral photography}

Apart from camera, lens, and flash, there are few accessories which are needed to take a high-resolution image. These accessories include photographic mirrors, contractors, and retractors.

\section{Photographic mirror}

These are available in different sizes and have different coatings like rhodium and aluminum. Rhodium mirrors are recommended for dental photography (Figure 4). They are used to click a photograph of occlusal view, the palatal surface of maxillary anterior teeth, lateral view of the entire arch,etc. Placement of mirror to includethe desired area should be in such a way that it should not be uncomfortable for the patient and, unnecessary area (retractors or nose) should not be captured in the image. To avoid fogging of these mirrors while using, one should pass a mirror through a hot water tap and to clean these mirrors, we can use paper towels without causing damage. ${ }^{19,20}$

\section{Retractors}

Retractors are available in different sizes and materials (plastic and metal). Plastic retractors are recommended due to more flexibility than metal retractors (Figure 5). These retractors are autoclave able,but due to repeated autoclaving, it becomes cloudy over a period. It is an accessory which is used to retract the cheeksfor taking an image of a full arch (frontal and lateral view) and occlusal view. For easy placement and comfort for the patient, retractors should be moistened before use..$^{19,20}$

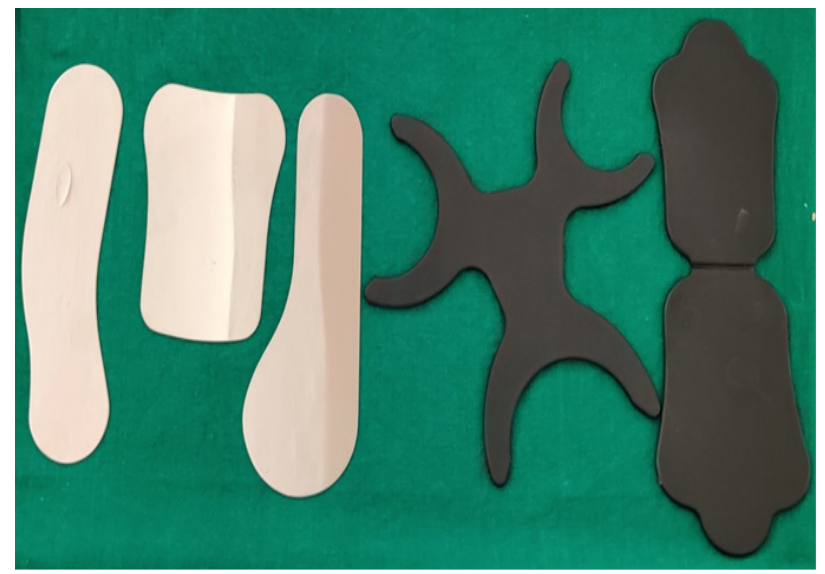

Figure 4 Different size and shapes of photographic mirror (left) and contratsers (right).

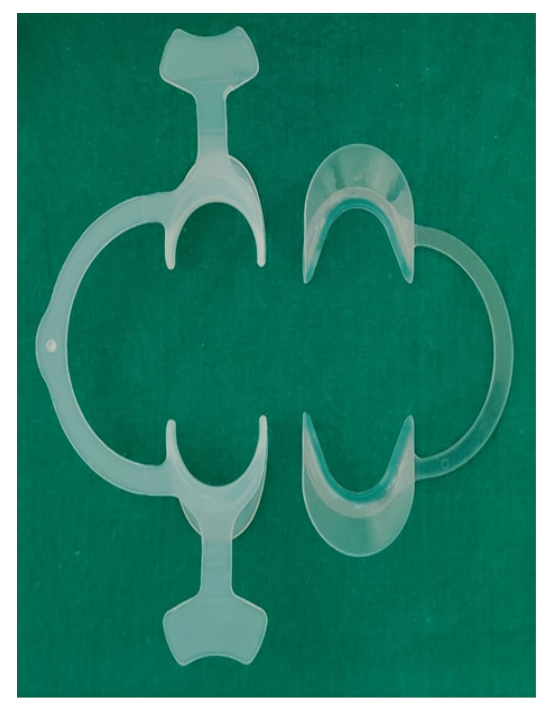

Figure 5 Different size of cheek retractors. 


\section{Contractors}

The contraster is used to create a black background to capture the images of anterior teeth. It enhances the transparency of incisal edges and blocks the structures of the oral cavity behind the teeth (Figure 4). These contractors are made up of black anodized aluminium or bendable copper sheet covered with silicone rubber and are available in different shapes. ${ }^{21}$

\section{Conclusion}

In dentistry, photography has become a part of the treatment. It is the best communication tool for better diagnosis, treatment planning, and even for education purpose. A digital camera with a $100 \mathrm{~mm}$ lens and manual mode for almost all features are recommended for dentistry. Knowledge of camera and specific protocols while taking a photograph enhances the quality of clinical photography. Goodphotography requires a skill of the operator,and it is timeconsuming to set a camera in every case according to the surrounding condition. Hence, in the future development of a camera which modifies all the setting based on surrounding condition by itself with a better quality of an image is recommended.

\section{Acknowledgments}

None.

\section{Conflicts of interest}

The author declares that there is no conflict of interest to disclose.

\section{Funding}

None.

\section{References}

1. Kalpana D, Rao SJ, Joseph JK, et al. Digital dental photography. Indian J Dent Res. 2018;29(4):507-512.

2. Galante DL. History and current use of clinical photography in orthodontics. J Calif Dent Assoc. 2009;37(3):173-174.

3. Griffin JD. Using digital photography to visualize, plan, and prepare a complex porcelain veneer case. Pract Proced Aesthet Dent. 2008;20(1):3945 .

4. American Academy of Cosmetic Dentistry. Photographic documentation and evaluation in cosmetic dentistry: a guide to accreditation photography. Madison, USA. 2009:16.
5. Zyman P, Etienne JM. Recording and communicating shade with digital photography: concepts and considerations. Pract Proced Aesthet Dent. 2002;14(1):49-51.

6. Swift JE, Quroz L, Hall SA. An introduction to clinical dental photography. Quintessence Int. 1987;18(12):859-869.

7. Snow SR. Dental photography systems: required features for equipment selection. Compend Contin Educ Dent. 2005;26(5):309-310.

8. Fahim R, Thakur R. Digital dental photography: The guidelines for a practical approach. TMU J Dent. 2014;1(3):106-112.

9. Ahmad I. Digital and Conventional Dental Photography: A Practical Clinical Manual. Quintessence Publishing Co Inc., USA 2004:1-229.

10. Sreevatsan R, Philip K, Peter E, et al. Digital photography in general and clinical dentistry-technical aspects and accessories. International Dental Journal of Students Research. 2015;3(1):17-24.

11. Ahmad I. Digital dental photography. Part 5: lighting. British dental journal. 2009;207(1):13-18.

12. Ahmad I. Digital dental photography. Part 4: choosing a camera. British dental journal. 2009;206(11):575-581.

13. Terry DA, Snow SR, McLaren EA. CE 1-Contemporary Dental Photography: Selection and Application. Compend Contin Educ Dent. 2008;29(8):432-436.

14. Ahmad I. Digital dental photography. Part 6: Camera setting. Br Dent J. 2009;207(2):63-69.

15. Snow SR. Assessing and achieving accuracy in digital dental photography. $J$ Calif Dent Assoc. 2009;37(3):185-191.

16. Laws R. The author's guide to controlling the photograph. Journal of Prosthetic Dentistry. 2001;85(3):213-218.

17. Bengel W. Mastering digital dental photography. Quintessence Publishing Co Ltd., UK. 2006:394.

18. Bengel WM. Digital photography and the assessment of therapeutic results after bleaching procedures. J Esthet Restor Dent. 2003;15(Suppl 1):S21-S32.

19. Vargas MA. Maxillary and mandibular occlusal photographs. $J$ Prosthodont. 2003;12(2):149-151.

20. Vargas MA, Lloyd PM. Maxillary and mandibular lateral view photographs. J Prosthodont. 2003;12(3):227-229.

21. Vargas MA. Photographing a view of the maxillary anterior teeth with a black background. J Prosthodont. 2002;11(3):208-210. 